\title{
Precision temperature sensing in the presence of magnetic field noise and vice-versa using nitrogen-vacancy centers in diamond
}

Wojciechowski, Adam M.; Karadas, Mürsel; Osterkamp, Christian; Jankuhn, Steffen; Meijer, Jan; Jelezko, Fedor; Huck, Alexander; Andersen, Ulrik Lund

Published in:

Applied Physics Letters

Link to article, DOI:

$10.1063 / 1.5026678$

Publication date:

2018

Document Version

Publisher's PDF, also known as Version of record

Link back to DTU Orbit

Citation (APA):

Wojciechowski, A. M., Karadas, M., Osterkamp, C., Jankuhn, S., Meijer, J., Jelezko, F., Huck, A., \& Andersen, U. L. (2018). Precision temperature sensing in the presence of magnetic field noise and vice-versa using nitrogen-vacancy centers in diamond. Applied Physics Letters, 113(1), [013502].

https://doi.org/10.1063/1.5026678

\section{General rights}

Copyright and moral rights for the publications made accessible in the public portal are retained by the authors and/or other copyright owners and it is a condition of accessing publications that users recognise and abide by the legal requirements associated with these rights.

- Users may download and print one copy of any publication from the public portal for the purpose of private study or research.

- You may not further distribute the material or use it for any profit-making activity or commercial gain

- You may freely distribute the URL identifying the publication in the public portal 


\section{Precision temperature sensing in the presence of magnetic field noise and vice-versa using nitrogen-vacancy centers in diamond}

Adam M. Wojciechowski, Mürsel Karadas, Christian Osterkamp, Steffen Jankuhn, Jan Meijer, Fedor Jelezko, Alexander Huck, and Ulrik L. Andersen

Citation: Appl. Phys. Lett. 113, 013502 (2018); doi: 10.1063/1.5026678

View online: https://doi.org/10.1063/1.5026678

View Table of Contents: http://aip.scitation.org/toc/apl/113/1

Published by the American Institute of Physics

\section{Articles you may be interested in}

Artificial electronic synapse characteristics of a Ta/Ta2 $\mathrm{O}_{5-\mathrm{x}} / \mathrm{Al}_{2} \mathrm{O}_{3} / \mathrm{InGaZnO}_{4}$ memristor device on flexible stainless steel substrate

Applied Physics Letters 113, 013503 (2018); 10.1063/1.5027776

Hybrid energy harvesting from mechanical vibrations and magnetic field

Applied Physics Letters 113, 013901 (2018); 10.1063/1.5038412

Entanglement control and magic angles for acceptor qubits in $\mathrm{Si}$

Applied Physics Letters 113, 012102 (2018); 10.1063/1.5036521

Control of dipole properties in high-k and $\mathrm{SiO}_{2}$ stacks on $\mathrm{Si}$ substrates with tricolor superstructure

Applied Physics Letters 113, 012103 (2018); 10.1063/1.5034494

Ultrathin amorphous $\mathrm{ZnGe}$ SnO films for high performance ultra-thin-film transistors

Applied Physics Letters 113, 013504 (2018); 10.1063/1.5031448

Multi-color and multidirectional-steerable Smith-Purcell radiation from 2D sub-wavelength hole arrays Applied Physics Letters 113, 013501 (2018); 10.1063/1.5034248

\section{Conference Proceedings}




\title{
Precision temperature sensing in the presence of magnetic field noise and vice-versa using nitrogen-vacancy centers in diamond
}

\author{
Adam M. Wojciechowski, ${ }^{1,2}$ Mürsel Karadas, ${ }^{3}$ Christian Osterkamp, ${ }^{4}$ Steffen Jankuhn, ${ }^{5}$ \\ Jan Meijer, ${ }^{5}$ Fedor Jelezko, ${ }^{4}$ Alexander Huck, ${ }^{1}$ and Ulrik L. Andersen ${ }^{1}$ \\ ${ }^{1}$ Center for Macroscopic Quantum States (bigQ), Department of Physics, Technical University of Denmark, \\ Fysikvej 309, 2800 Kgs. Lyngby, Denmark \\ ${ }^{2}$ Institute of Physics, Jagiellonian University, Łojasiewicza 11, 30-363 Kraków, Poland \\ ${ }^{3}$ Department of Electrical Engineering, Technical University of Denmark, Ørsteds Plads, 2800 Kgs. Lyngby, \\ Denmark \\ ${ }^{4}$ Institute for Quantum Optics and Center for Integrated Quantum Science and Technology (IQST), \\ Ulm University, Albert-Einstein-Allee 11,89081 Ulm, Germany \\ ${ }^{5}$ Felix Bloch Institute for Solid State Physics, University of Leipzig, 04103 Leipzig, Germany
}

(Received 22 February 2018; accepted 15 June 2018; published online 3 July 2018)

\begin{abstract}
We demonstrate a technique for precision sensing of the temperature or the magnetic field by simultaneously driving two hyperfine transitions involving distinct electronic states of the nitrogenvacancy center in diamond. Frequency modulation of both driving fields is used with either the same or opposite phase, resulting in the immunity to fluctuations in either the magnetic field or the temperature, respectively. In this way, a sensitivity of $1.4 \mathrm{nT} \mathrm{Hz}^{-1 / 2}$ or $430 \mu \mathrm{K} \mathrm{Hz}^{-1 / 2}$ is demonstrated. The presented technique only requires a single frequency demodulator and enables the use of phase-sensitive camera imaging sensors. A simple extension of the method utilizing two demodulators allows for simultaneous, independent, and high-bandwidth monitoring of both the magnetic field and the temperature. Published by AIP Publishing. https://doi.org/10.1063/1.5026678
\end{abstract}

Negatively charged nitrogen-vacancy (NV) color centers $^{1,2}$ have become a popular tool for precision magneticfield sensing at the nano- to milli-meter length scales, ${ }^{3-6}$ with the dc sensitivity in the tens of $\mathrm{pT} / \mathrm{Hz}^{1 / 2}$ range $^{7}$ and even higher ac sensitivities. ${ }^{8}$ At room temperature, the energy-level structure of the NV ground state is also sensitive to temperature fluctuations; $;^{9,10}$ a temperature change of $1 \mathrm{mK}$ causes frequency shifts equivalent to a few-nT magnetic field change. Thus, the presence of noise in one of these quantities may have an impact on the precision of measuring the other quantity, unless there is a way of discerning them, for example, by temporal signatures.

The temperature of the diamond can be easily read out from the optically detected magnetic resonance (ODMR) spectrum of the NV fluorescence by sweeping a microwave (MW) frequency of around $2.8 \mathrm{GHz}$. The temperature is then inferred from the positions of two opposite spin transitions corresponding to the same crystallographic orientation. ${ }^{9}$ Thermometry using pulsed MW protocols or $c w$-ODMR has been demonstrated for single NVs, nanodiamonds and bulk samples. ${ }^{11-14}$ So far, temperature sensing was demonstrated under stationary or slowly varying conditions at time scales of many seconds to hours. Large-range $( \pm 100 \mathrm{~K})$ and high bandwidth temperature sensing has been shown in Ref. 15 requiring, however, averaging of thousands of measurements. This approach is therefore only suitable for the measurement of well-controlled transients.

In this article, we report on a method for recording temperature transients on millisecond time scales in a single-shot measurement, while being immune to a magnetic field that induces comparable resonance shifts. The scheme can also be reversed in order to record magnetic signals that are immune to temperature variations. Such temperature transients may be due to laser and/or MW signals operated in a quasi-continuous mode. Similar concepts have been introduced for pulsed MW schemes, a magnetometer immune to temperature drifts ${ }^{16}$ and a thermometer insensitive to magnetic fields. ${ }^{11}$

Our approach relies on the simultaneous driving of two transitions $\left(m_{\mathrm{S}}=0 \leftrightarrow m_{\mathrm{S}}= \pm 1\right)$ using $\mathrm{cw}$, frequency- or amplitude-modulated MW fields with either the same or the opposite phase of modulation. The common-mode shift of the resonance frequencies with the temperature and their differential shift with magnetic field change are used for generating the signal of interest from the ODMR spectrum. Depending on the choice of electronic transitions and modulation phases (polarity of each contribution), the output signal may contain information about the temperature, the magnetic field or both. Interference effects that occur due to the simultaneous driving of transitions that share one substate $\left(m_{\mathrm{S}}=0\right)^{17,18}$ are avoided here by addressing distinct and well resolved hyperfine transitions [Fig. 1(a)].

We use a [100]-oriented, $2 \times 2 \times 0.5 \mathrm{~mm}^{3}$ ultrapure diamond crystal ([N] $<1 \mathrm{ppb})$. The diamond was overgrown in a chemical vapor deposition process with an approximately $1 \mu \mathrm{m}$ thin, isotopically purified $\left(\left[{ }^{12} \mathrm{C}\right]>99.99 \%\right)$ layer, doped with ${ }^{15} \mathrm{~N}\left(\left[{ }^{15} \mathrm{~N}\right] \sim 10 \mathrm{ppm}\right)$. In order to introduce vacancies, the sample was implanted with $1.8 \mathrm{MeV}$ helium ions with a dose of $10^{15} \mathrm{~cm}^{-2}$. The NV concentration after $2 \mathrm{~h}$ of annealing at $900{ }^{\circ} \mathrm{C}$ is estimated to be on the order of 0.1-1 ppm.

The diamond sample was glued with silicone into a 3Dprinted holder placed on top of an inverted microscope setup together with a MW antenna structure of a design similar to that in Ref. 19. The NV fluorescence was collected by a NA $=0.7$ objective (Mitutoyo M Plan Apo NIR HR) and imaged $(30 \times$ magnification) onto a biased photodetector (Thorlabs 
(a)

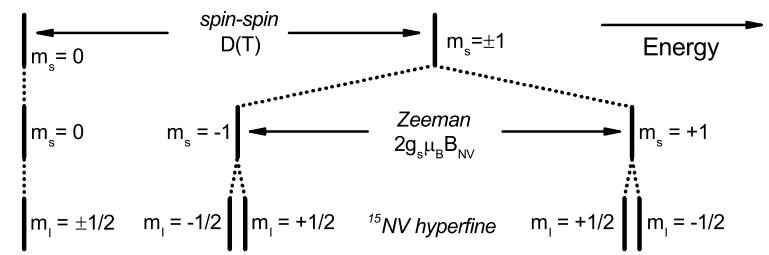

(b)

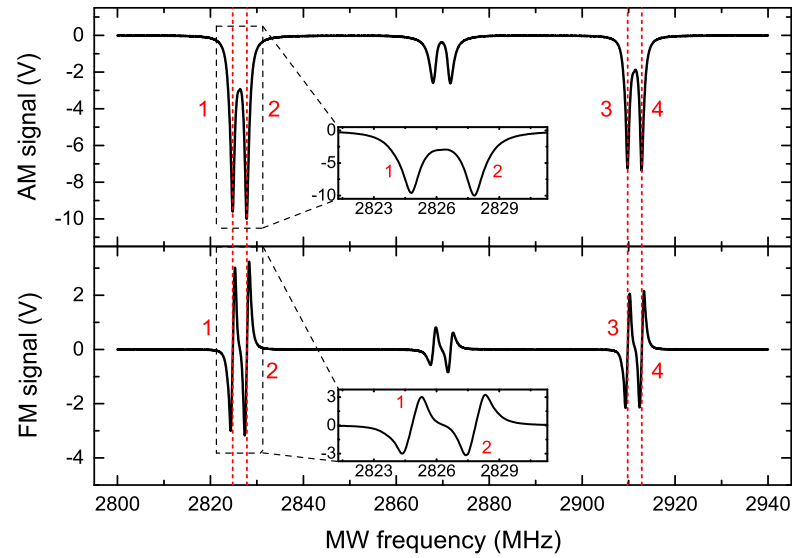

FIG. 1. (a) NV ground state level diagram indicating three types of interactions influencing the energies. The hyperfine peak separation for ${ }^{15} \mathrm{NV}$ is 3.05 MHz. (b) ODMR spectra recorded with an amplitude (top) and a frequency (bottom) modulated microwave field in a [110]-oriented bias magnetic field. Vertical dashed lines indicate MW frequencies which are also labeled by the red numbers. The slight asymmetry between the left and right pairs of resonances results from the finite MW antenna bandwidth. The peak depth for AM corresponds to 3\% fluorescence contrast.

DET36A). The bottom diamond surface has been antireflection coated with $\mathrm{SiO}_{2}(95 \mathrm{~nm})$, while the top surface was coated with $\mathrm{Al}(300 \mathrm{~nm})$ in order to reflect the fluorescence and excitation light and prevent it from passing through the samples we intend to place on top of the diamond in future experiments. Green laser light at $532 \mathrm{~nm}$ was sent through an acousto-optical modulator (AOM, Isomet M1133-aQ110-1V) and focused on the back focal plane of the objective, resulting in a wide-field $(\sim 80 \mu \mathrm{m}$ spot diameter) illumination with $150 \mathrm{~mW}$ of laser power reaching the diamond. Additional laser light of around $75 \mathrm{~mW}$ of power was used for quick heating of the diamond surface. It was derived from the same laser and sent through an independent AOM to the top surface of the diamond, which was painted with a red marker to enhance the beam absorption.

The outputs of two MW generators [Stanford Research Systems (SRS) SG394] were combined in a power combiner [Mini-Circuits (M-C) ZFRSC-183-S+] and sent through a switch (M-C ZASWA-2-50DR+) to a high-power amplifier (M-C ZHL-16W-43+). Its output is connected to the MW antenna through a 3-port circulator (MECA CS-3.000), which allows for monitoring the reflected power. An external function generator (Rigol DG1022) is used to generate the FM or AM signal for each MW source, providing independent control of the modulation parameters. The photodetector is connected to the current input of a lock-in amplifier (SRS SR850, $10^{6}$ transimpedance gain) which provides phase-sensitive demodulation of the fluorescent signal. All data have been recorded with a $40 \mathrm{kHz}$ sine-wave modulation frequency and a $100 \%$ depth (for $\mathrm{AM}$ ) or $\pm 500 \mathrm{kHz}$ frequency deviation (for FM), respectively. Lock-in time constants of either $100 \mu$ s or $1 \mathrm{~ms}$ were used, and the filter roll-off was set to $18 \mathrm{~dB} /$ octave corresponding to around $1 \mathrm{kHz}$ or $100 \mathrm{~Hz}$ bandwidth, respectively. A bias magnetic field of around $1.9 \mathrm{mT}$ was aligned in-plane with the diamond surface along the [110] direction. An additional set of coils in a near-Helmholtz configuration allowed for fine-tuning of the field alignment and for the application of additional magnetic fields controlled by a data acquisition card. In this geometry, the NV center spin resonance frequencies are sensitive to the magnetic field component parallel to the diamond surface. The resonance position shifts by $\approx 22.86 \mathrm{~Hz} / \mathrm{nT}$ resulting from the geometric factor of $\cos \left(90^{\circ}-\theta_{B} / 2\right)=\sqrt{2 / 3}$, where $\theta_{B}$ $\approx 109.5^{\circ}$ is the bond angle in diamond. Our experiments were performed at room temperature resulting in temperatureinduced shifts of $\left.\frac{\partial D}{\partial T}\right|_{25^{\circ}} \sim 75 \mathrm{~Hz} / \mathrm{mK},{ }^{9,12,16,20}$ where $D$ indicates the zero-magnetic field splitting parameter.

Figure 1(b) shows the $c w$-ODMR spectra recorded with a single MW source for two types of modulation. The outermost pairs of resonances are formed by degenerate pairs of hyperfine transitions belonging to the two distinct crystallographic directions. This allowed us to record twice the fluorescence contrast, at the cost of magnetic shifts being reduced by a factor of $\sqrt{2 / 3}$.

From the $c w$-ODMR spectra, one can easily determine the magnetic field value by measuring the frequency difference between an appropriate transition pair, for example, 1 and 3 in Fig. 1. For small magnetic field changes causing frequency shifts less than the resonance linewidth, a high bandwidth readout is possible. This can be accomplished by tuning the MW frequency, $f$, to the center (FM) or side (AM) of the resonance, where the signal, $S$, has the steepest and approximately linear spectral dependence. The signal detected in-phase with the modulation reference can be expressed as

$$
\left.S \propto \frac{\partial U}{\partial B}\right|_{f} \Delta B+\left.\frac{\partial U}{\partial T}\right|_{f} \Delta T
$$

where $\mathrm{U}$ is the ODMR voltage in Fig. 1 and $\Delta$ denotes the change in the parameter. The last term in 1 is often neglected as the temperature variations typically occur on much longer time scales than magnetic signals of interest. In our experiments, we are using a quasi- $c w$ timing protocol where light and MWs are switched on with a low duty-cycle (interrogation time of $200 \mathrm{~ms}$, repeated every $10 \mathrm{~s}$ ) in order to limit the heating of the diamond and possible interactions with samples we intend to place on its surface in planned experiments. This results in a periodic diamond warm-up, in which case temperature variations can no longer be neglected.

Simultaneous driving of several hyperfine transitions, for example those labeled 1 and 2 in Fig. 1(b), is often used to enhance the sensitivity of the diamond probe because this yields an increase in the fluorescence contrast. ${ }^{7,21}$ For ${ }^{15} \mathrm{NV}$, a double drive may be used and the signal is then given by

$$
S \propto\left(\left.\frac{\partial U}{\partial B}\right|_{f_{1}, \phi_{1}}+\left.\frac{\partial U}{\partial B}\right|_{f_{2}, \phi_{2}}\right) \Delta B+\left(\left.\frac{\partial U}{\partial T}\right|_{f_{1}, \phi_{1}}+\left.\frac{\partial U}{\partial T}\right|_{f_{2}, \phi_{2}}\right) \Delta T,
$$

where we have explicitly indicated the modulation phase $\phi_{i}$ of the $i$-th MW driving field with respect to the lock-in 
reference. In the following, we restrict the modulation phase to be either $0^{\circ}$ or $180^{\circ}$ resulting only in a change of sign in appropriate partial derivatives.

A typical experimental protocol and the corresponding lock-in output signals are shown in Fig. 2. The top panel illustrates the pulse sequence that is repeated every $10 \mathrm{~s}$. The laser light and MW fields are switched on for $200 \mathrm{~ms}$, starting at $t=20 \mathrm{~ms}$. Two additional field pulses are applied during the measurement. The heating beam is applied between 100 and $150 \mathrm{~ms}$ and a $100 \mathrm{nT}$ magnetic field is switched on between 160 and $200 \mathrm{~ms}$. The solid black curve in the bottom part of Fig. 2 shows the lock-in response when the resonances labeled 1 and 2 (c.f. Fig. 1) are driven with an identical FM modulation with $\phi_{1}=\phi_{2}=0$. We neglect the shaded area in the further analysis as it corresponds to the time when no excitation light is present and a short duration (between 20 and $30 \mathrm{~ms}$ ) of lock-in recovery after switching the laser and MW fields. The central part of the curve shows an oscillation at $50 \mathrm{~Hz}$ due to magnetic-field noise in the laboratory and a temperature transient visible as a skew of the oscillating signal. When driving a pair of hyperfine transitions belonging to the same electron-spin states with inphase modulations, the right-hand side in Eq. (2) simplifies to twice that of a single-drive case described by Eq. (1).

The situation changes dramatically when two distinct electron transitions (for example, resonances labeled 2 and 4) are driven with the same modulation. The energy derivative on the magnetic field is opposite for the $m_{\mathrm{S}}= \pm 1$ states, and therefore $\left.(\partial U / \partial B)\right|_{f 1}=-\left.(\partial U / \partial B)\right|_{f 2}$. Thus, the magnetic-field dependent terms in Eq. (2) cancel out and the recorded signal only carries information on the diamond temperature, which is plotted as a dashed red curve in Fig. 2. Reversing the modulation phase by $180^{\circ}$ on one MW source leads to a simultaneous sign change of both terms at its frequency in Eq. (2). A common-mode energy change due to temperature variation is then canceled out and only a differential shift due to the magnetic-field is recorded. This is

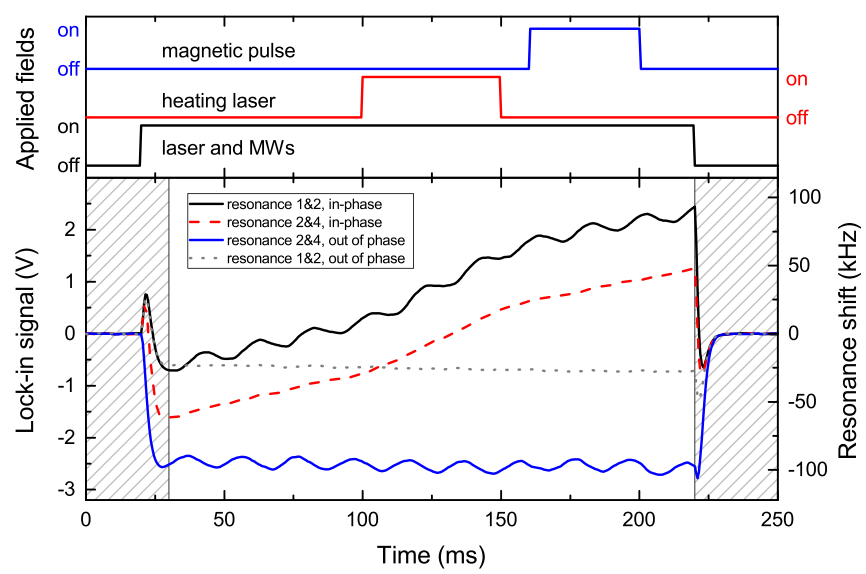

FIG. 2. Top panel: timing diagram showing the switching of laser and MW fields and the applied heating and magnetic field pulses. The sequence is repeated every $10 \mathrm{~s}$. Bottom panel: Single-shot transient recordings acquired in four distinct configurations: with MW sources tuned to resonances labeled 1 and 2 or 2 and 4 (see Fig. 1), and their frequency modulation having either identical or opposite phases. Recorded signals reflect changes in the magnetic field (solid blue), the temperature (dashed red), both at once (solid black) or neither of those (dashed). The shaded areas indicate time windows when no sensing was performed. The lock-in time constant was set to $1 \mathrm{~ms}$. plotted as the solid blue curve in Fig. 2. The remaining fourth combination of driving resonances 1 and 2 with outof-phase modulated sources leads to a signal that is immune to both temperature and magnetic-field variations, which is plotted as the gray dotted curve.

In order to further support the attribution of frequency shifts to in- and out-of-phase drives of resonances 2 and 4, we analyze those signals in more detail in Fig. 3. Figure 3(a) illustrates that the magnetic signal (blue curve) has a dominant component oscillating at the mains frequency of $50 \mathrm{~Hz}$ with a peak-to-peak amplitude of 392(4) nT. This signal occurs due to the stray laboratory fields and also contains higher harmonic components. The observation of the applied $100 \mathrm{nT}$ field step (black dashed trace) is hindered by the mains hum and can be seen clearly after subtraction of the latter, as shown by the red curve. The temperature transients recorded with and without the heating pulse are shown in Fig. 3(b). These transients exhibit $\sim 1$ and $1.5 \mathrm{~K}$ change occurring over the $200 \mathrm{~ms}$ interaction time with the auxiliary heating beam being off (gray trace) and on (red dashed trace), respectively. Without the heating pulse, the dominant heating mechanism is the absorption of green excitation laser light by the aluminum layer. The power reflection coefficient for aluminum on diamond is around $83 \%$ for normal incidence and the remaining part is absorbed. Assuming that 25 $\mathrm{mW}$ of optical power is absorbed in the Al coating, the expected heating rate for a thermally isolated diamond is $\approx 6.9 \mathrm{~K} / \mathrm{s}$. Shortly after switching on the laser, the actual diamond heating rate is $5.9(1) \mathrm{K} / \mathrm{s}$, which is in quantitative agreement with the estimated value considering that the heat capacity of the silicone layer surrounding the diamond is negligible. At longer times, the heat transfer to the holder causes a slow leveling-off of the temperature transient. The presence of the additional heating pulse increases the heating rate to $12.9(1) \mathrm{K} / \mathrm{s}$ for the duration of the pulse, which

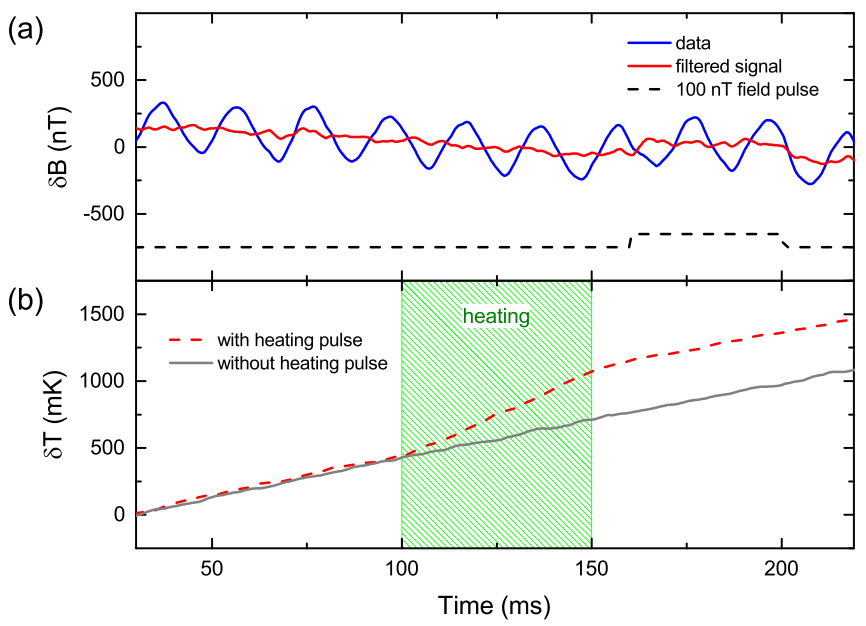

FIG. 3. Relative magnetic field (solid blue) and temperature (dashed red) changes extracted from Fig. 2: (a) the response to the change in the magnetic field. The applied field step is hindered by large $\left(\sim 400 \mathrm{nT}_{p-p}\right)$ oscillations at the mains frequency and recovered after subtraction of the $50 \mathrm{~Hz}$ component (solid red); (b) a comparison of temperature transients recorded with and without the heating pulse. An additional laser, when on, increases the heating rate from $5.9 \mathrm{~K} / \mathrm{s}$ to $12.9 \mathrm{~K} / \mathrm{s}$, as verified by the heating period in the range $100-150 \mathrm{~ms}$. 
corresponds to approximately one half of the rate expected from the power impeding on the painted surface.

In order to characterize the sensitivity of our setup to the temperature and the magnetic field, we have recorded signals without pulsing the laser or MWs. Time traces of the lock-in output with the length of $1 \mathrm{~s}$ were recorded and a root-meansquare amplitude spectral density was calculated using the Hanning window function. Spectra from 50 traces were subsequently rms averaged and the resulting data are plotted in Fig. 4. In the magnetically sensitive configuration (black trace), distinct peaks are visible at the first, second, third and fifth harmonics of the $50 \mathrm{~Hz}$ mains frequency. Additionally, an applied $20 \mathrm{nT}_{\mathrm{p}-\mathrm{p}}$ sine-wave signal is visible at $40 \mathrm{~Hz}$, which serves as a calibration field. The level of the noise floor is $\sim 1.4 \mathrm{nT} \mathrm{Hz}^{-1 / 2}$ up to the lock-in filter roll-off frequency of $\sim 1 \mathrm{kHz}$. In the temperature-sensitive mode (red trace), the response to magnetic fields vanishes and a temperature sensitivity of $\sim 430 \mu \mathrm{K} \mathrm{Hz}^{-1 / 2}$ is achieved. All recordings show a small peak around $67 \mathrm{~Hz}$, which we attribute to electronic pick-up. The noise floor is about 3 times higher than the optical shot-noise level for the detected fluorescence and mainly originates from the electronic noise in our detection system.

Similarly to the frequency modulation of MW sources, $\mathrm{AM}$ also provides a way of differential- or common-mode signal cancellation (via slope-polarity change) by tuning the MW frequency to either side of an appropriate resonance. However, in our experiment, we have found that FM signals are more immune to laser intensity noise since the highest sensitivity is achieved exactly on-resonance where the signal output is close to zero. Our method can also be extended to the case of independent modulation of MW sources with two distinct frequencies. The photocurrent can then be demodulated by two lock-in amplifiers and simultaneously provide information about both the magnetic-field and temperature. On the other hand, a single demodulator approach as

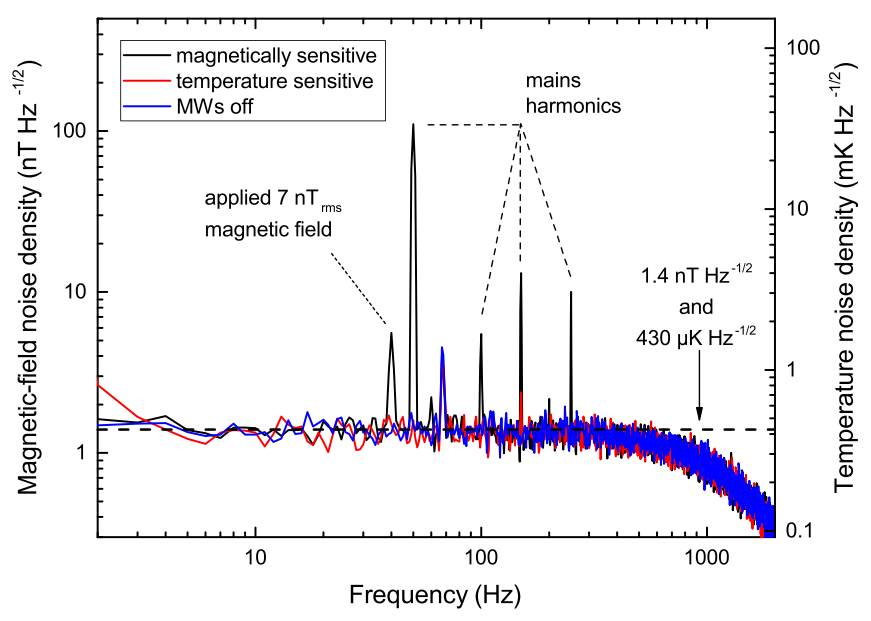

FIG. 4. Recorded noise spectral density in the units of the magnetic field and temperature, respectively, with MWs tuned to resonances 1 and 3 (see Fig. 1). The magnetic signal corresponds to opposite modulation phases and shows peaks at the mains harmonics. The temperature signal is recorded with identical phase modulations and is virtually identical to the noise floor recorded in the absence of MWs. The signal roll-off around $1 \mathrm{kHz}$ is due to the lock-in filtering with a $100 \mu$ s time constant. Discrete peaks are broadened and have their amplitudes reduced by a factor of 0.8 due to the windowing function. presented here can be combined with a phase-sensitive camera sensor for wide-field sensing. ${ }^{22}$ In such a camera, the demodulation is performed on a per-pixel basis and is limited to a single frequency for currently available devices.

In conclusion, we have demonstrated a MW modulation scheme for $c w$-ODMR that allows for monitoring temperature or magnetic field variations hindered by the presence of a large noise (or signal) in the other modality. Our method is based on in- and out-of-phase modulations of two MW fields driving distinct spin transitions in the NV ground state. It allows for precision temperature sensing applications with sub-millikelvin resolution and up to $10 \mathrm{~K}$ of dynamic range. The sensitivity demonstrated in this work is limited by the amount of fluorescence light and can be further increased using thicker sensing layers. With the current setup, we have achieved three orders of magnitude better temperature sensitivity than in Ref. 15 and demonstrated single-shot transient recording on a millisecond time scale. We anticipate that this technique may find applications in micro-calorimetry for chemical reaction studies. The advantage over previously demonstrated ODMR-based thermometry ${ }^{14,17}$ stems from magnetic noise rejection and simultaneous, rather than consecutive, tracking of two resonance shifts. The latter results in a high bandwidth of $1-10 \mathrm{kHz}$ and the ability to record the signal of interest in a single-shot using a single lock-in demodulator. Additionally, the proposed scheme is well suited for magnetic field recording immune to the presence of thermal transients. This may be important for magnetic studies of biological systems, where continuous exposure to high-power electro-magnetic fields can cause physiological signal deterioration and has to be avoided. The on-off keying of MW and/or laser fields with a low duty-cycle may be used to limit the mean power delivered to the sample in which case our technique can alleviate the accompanying effect of temperature transients induced by field toggling. Finally, the presented scheme may be easily extended to a highbandwidth thermal or magnetic imaging scenario using a lock-in camera for wide-field fluorescent light detection.

We thank Kaare Hartvig Jensen for 3D printing of the prototype diamond holder and Kristian Haagsted Rasmusen for coating the diamond. This work was supported by the Innovation Fund Denmark (through the EXMAD and QUBIZ projects), Novo Nordisk Foundation (NNF16OC0023514), IQst, DFG, VW Stiftung, ERC, BMBF, and EU DIADEMS.

${ }^{1}$ F. Jelezko, I. Popa, A. Gruber, C. Tietz, J. Wrachtrup, A. Nizovtsev, and S. Kilin, Appl. Phys. Lett. 81, 2160 (2002).

${ }^{2}$ M. W. Doherty, N. B. Manson, P. Delaney, F. Jelezko, J. Wrachtrup, and L. C. L. Hollenberg, Phys. Rep. 528, 1 (2013).

${ }^{3}$ J. R. Maze, P. L. Stanwix, J. S. Hodges, S. Hong, J. M. Taylor, P. Cappellaro, L. Jiang, M. V. G. Dutt, E. Togan, A. S. Zibrov, A. Yacoby, R. L. Walsworth, and M. D. Lukin, Nature 455, 644 (2008).

${ }^{4}$ G. Balasubramanian, I. Y. Chan, R. Kolesov, M. Al-Hmoud, J. Tisler, C. Shin, C. Kim, A. Wojcik, P. R. Hemmer, A. Krueger, T. Hanke, A. Leitenstorfer, R. Bratschitsch, F. Jelezko, and J. Wrachtrup, Nature 455, 648 (2008).

${ }^{5}$ L. Rondin, J.-P. Tetienne, T. Hingant, J.-F. Roch, P. Maletinsky, and V. Jacques, Rep. Prog. Phys. 77, 056503 (2014).

${ }^{6}$ R. Schirhagl, K. Chang, M. Loretz, and C. L. Degen, Annu. Rev. Phys. Chem. 65, 83 (2014). 
${ }^{7}$ J. F. Barry, M. J. Turner, J. M. Schloss, D. R. Glenn, Y. Song, M. D. Lukin, H. Park, and R. L. Walsworth, Proc. Natl. Acad. Sci. 113, 14133 (2016).

${ }^{8}$ T. Wolf, P. Neumann, K. Nakamura, H. Sumiya, T. Ohshima, J. Isoya, and J. Wrachtrup, Phys. Rev. X 5, 041001 (2015)

${ }^{9}$ V. M. Acosta, E. Bauch, M. P. Ledbetter, A. Waxman, L. S. Bouchard, and D. Budker, Phys. Rev. Lett. 104, 070801 (2010).

${ }^{10}$ M. W. Doherty, V. M. Acosta, A. Jarmola, M. S. J. Barson, N. B. Manson, D. Budker, and L. C. L. Hollenberg, Phys. Rev. B 90, 041201 (2014).

${ }^{11}$ D. M. Toyli, C. F. de las Casas, D. J. Christle, V. V. Dobrovitski, and D. D. Awschalom, Proc. Natl. Acad. Sci. 110, 8417 (2013).

${ }^{12}$ P. Neumann, I. Jakobi, F. Dolde, C. Burk, R. Reuter, G. Waldherr, J. Honert, T. Wolf, A. Brunner, J. H. Shim, D. Suter, H. Sumiya, J. Isoya, and J. Wrachtrup, Nano Lett. 13, 2738 (2013).

${ }^{13}$ G. Kucsko, P. C. Maurer, N. Y. Yao, M. Kubo, H. J. Noh, P. K. Lo, H. Park, and M. D. Lukin, Nature 500, 54 (2013).

${ }^{14}$ H. Clevenson, M. E. Trusheim, C. Teale, T. Schröder, D. Braje, and D. Englund, Nat. Phys. 11, 393 (2015)
${ }^{15}$ Y.-K. Tzeng, P.-C. Tsai, H.-Y. Liu, O. Y. Chen, H. Hsu, F.-G. Yee, M.-S. Chang, and H.-C. Chang, Nano Lett. 15, 3945 (2015).

${ }^{16}$ K. Fang, V. M. Acosta, C. Santori, Z. Huang, K. M. Itoh, H. Watanabe, S. Shikata, and R. G. Beausoleil, Phys. Rev. Lett. 110, 130802 (2013).

${ }^{17}$ P. Kehayias, M. Mrózek, V. M. Acosta, A. Jarmola, D. S. Rudnicki, R. Folman, W. Gawlik, and D. Budker, Phys. Rev. B 89, 245202 (2014).

${ }^{18}$ M. Mrozek, A. M. Wojciechowski, D. S. Rudnicki, J. Zachorowski, P. Kehayias, D. Budker, and W. Gawlik, Phys. Rev. B 94, 035204 (2016).

${ }^{19}$ K. Sasaki, Y. Monnai, S. Saijo, R. Fujita, H. Watanabe, J. Ishi-Hayase, K. M. Itoh, and E. Abe, Rev. Sci. Instrum. 87, 053904 (2016).

${ }^{20}$ X.-D. Chen, C.-H. Dong, F.-W. Sun, C.-L. Zou, J.-M. Cui, Z.-F. Han, and G.-C. Guo, Appl. Phys. Lett. 99, 161903 (2011).

${ }^{21}$ H. A. R. El-Ella, S. Ahmadi, A. M. Wojciechowski, A. Huck, and U. L. Andersen, Opt. Express 25, 14809 (2017).

${ }^{22}$ A. M. Wojciechowski, M. Karadas, A. Huck, C. Osterkamp, S. Jankuhn, J. Meijer, F. Jelezko, and U. L. Andersen, Rev. Sci. Instrum. 89, 031501 (2018). 